\title{
Menopausia: actitudes y conocimientos en un grupo de médicos internos, de la facultad de Medicina, de la Universidad de Antioquia
}

\author{
Ossa, JE*; Echeverry, JV; Penagos, GS; Gutiérrez, F; Uribe, F; Botero, JE; Céspedes, CM; Osorio, JW; Ríos, AP; \\ Ayora, MJ.
}

Recibido: Marzo 1/2000 - Revisado: Abril 3/2000

Aceptado: Junio 9/2000

\section{RESUMEN}

Se presenta los resultados de una encuesta realizada a los médicos internos de la Facultad de Medicina de la Universidad de Antioquia, en el segundo semestre de 1997. El proyecto se realizó con el doble propósito de construir conocimiento sobre el tema del climaterio femenino un tema determinado.

Se utilizó el censo y las técnicas de encuesta y escala Lickert para indagar sobre variables socioculturales, demográficas y de carácter científico/técnico. Se encontró que el nivel de conocimientos, medido por la "nota", fue de 2.85 + 0.83 ; la actitud frente a la terapia fue baja en el $44 \%$ y alta en el $40 \%$. Como se había previsto en la hipótesis, la relación entre actitudes y conocimientos fue directa, pero la asociación con variables sociodemográficas no pudo detetminarse debido a la homogenidad de la población.

PALABRAS CLAVES: Currículo, sexualidad, terapia de reemplazo

\section{SUMMARY}

This article reports the results of a survey carried out in the group of final-year medical students of the Shool of the University of Antioquia, en Medellín, Colombia, in the second semester of the year 1997. The aims of the study was, on the first hand, to help in the construction of a body of knowledge on the climacteric period in women, and additionally, to contribute to the goal of change of the medical curriculum in which the institution is involved. We departed from the hypothesis that the personal history of the individual limits the attitude and constrains the possibilities for the development of motivation and skills on a given subject.

The instrument was presented to all and each one of the students in the group and it consisted of a survey and a Licker's scale to search for in a scale from 0 to 5 , was $2.85+0.83$ while the attitude toward therapy was low in $44 \%$ a, and high in $40 \%$ of the students. The correlation between attitudes and knowledge was positive, but the demographic data was too homogeneous to allow any association to be made.

KEY WORDS: Curriculum, sexuality, replacement therapy

\section{Introducción}

El Climaterio femenino es un proceso, caracterizado por una serie de signos y síntomas orgánicos, secundarios a la disminución en el funcionamiento endocrino de los ovarios y por síntomas psíquicos relacionados con la historia personal y social de las mujecres. La cultura occidental se refiere peyorativamente a este período como "menopáusico", para relacionar el hecho biológico de la menopausia o última menstruación, que ocurre entre los 48 y 52 años, con el significante social de fin de la vida útil, pérdida de la capacidad reproductiva, subvaloración de la feminidad y la sexualidad y pérdida del estatus social. De esta manera, una etapa normal, en la que las mujeres viven aproximadamente el $33 \%$ de su vida, se

Grupo Biogénesis - Reproducción, Subgrupo de Bioantropología, Facultad de Medicina, Universidad de Antioquia, AA 1226 , Medellín, Colombia transforma en factor de riesgo social y familiar, para asegurar, de paso, la ideología reproductiva como el elemento de control de la vida de las mujeres.

Según la Organización Mundial de la Salud (OMS), a nivel mundial, la expectativa de vida ha aumentado entre 6 y 10 años; más para las mujeres que para los hombres, con un promedio de 74 años para los países industrializados y de 69 para los países en vía de desarrollo (1). En Colombia la población actual mayor de 65 años es del 3,9\% y en el 2.025 será del $9 \%(2,3)$, por lo cual es importante no sólo reconocer, prevenir y tratar la morbilidad física y sus factores de riesgo, sino también la morbilidad psíquica y social.

Las mujeres, en las dos últimas décadas, aún con mayores oportunidades de conocimientos, capacitación y acceso a funciones sociales representativas continúan manifestando actitudes negativas frente a la menopausia por la construcción social que se ha hecho de esta etapa de la vida. Los médicos, no siempre conscientes de que en su aprendizaje 
se perpetúan los mitos de la sociedad a la que pertenecen, también manifiestan actitudes negativas hacia el abordaje terapéutico integral de las mujeres en este proceso.

Actualmente está establecido que la terapia de reemplazo estrogénico a largo plazo, tiene efectos profilácticos benéficos en la osteoporosis, la enfermedad cardiovascular y la enfermedad de Alzheimer (4, 5, 6), a la vez que disminuye la mortalidad global durante el período de reemplazo (7) y mejora la calidad de vida. A pesar de estos conocimientos, la utilización de estrógenos por las mujeres en la postmenopausia sigue siendo bastante restringida. Menos del 20\% de este grupo de mujeres, en Estados Unidos, han sido prescritas con terapia de reemplazo y mucho menor es el número de mujeres que lo continúan recibiendo un año después de haberlo iniciado (8). En Inglaterra se demostró una utilización de hormonas de únicamente el $9 \%$ en mujeres entre los 60 y los 64 años de edad (9). En Colombia no disponemos en el presente de estudios al respecto, pero por la menor posibilidad de acceso poblacional a los sistemas de salud, nos atrevemos a suponer una utilización aún más baja de la terapia de reemplazo hormonal.

La baja prescripción de terapia de reemplazo hormonal, (TRH), se ha atribuido en los estudios ingleses, en gran medida, a concepciones erróneas de los médicos con respecto a la relación entre TRH y enfetmedad coronaria (10). Cerca de la mitad de los encuestados, en Inglaterra, considera que la TRH incrementa el riesgo de enfermedad cardíaca isquémica; incluso por parte de aquellos médicos que comprenden los beneficios a largo plazo existe un bajo nivel de prescripción; esto podría estar asociado con una actitud poco propicia por parte del médico asistente a informar, asesorar y tratar a la paciente sobre los efectos secundarios de la terapia, tales como: sangrado vaginal anormal o inesperado, sensibilidad mamaria, retención de líquidos o incremento de peso. También es necesario reconocer que en su preparación como estudiante, el médico tiene poca oportunidad de tratar el tema y menos de poner en práctica dicho aprendizaje pues éste, como otros temas de los contenidos curriculares, no son congruentes con las realidades epidemiológicas actuales.

En Colombia, un estudio realizado en 1996, por el Doctor Francisco Pardo en algunas Facultades de Medicina, encontró que son muy pocas las horas teóricas y prácticas asignadas al tema del climaterio; en horas teóricas, 6 Facultades asignan una hora y 2 Facultades asignan 2 horas; y, en horas prácticas, 2 Facultades asignan 16 horas, 2 Facultades dedican 6 horas y 3 Facultades no asignan tiempo a esta actividad. El tiempo físico dedicado no es necesariamente el mejor indicador, pero dentro de nuestra cultura universitaria esta situación sí resulta sospechosa. La pregunta es si la preparación que se está ofreciendo es suficiente para la medicina del futuro? (1).

Lo anterior nos ha llevado a proponer que si deseamos beneficiar un mayor número de mujeres en la posmenopausia con una prescripción adecuada y oportuna de la TRH, debemos empezar por jnvestigar cuáles son las actitudes y los conocimientos de nuestros médicos con respecto a la temática específica del climaterio femenino. En este contexto y con el ánimo de contribuir a la discusión que, sobre cambio curricular, se adelanta actualmente en la Universidad de Antioquia, iniciamos la presente investigación en el primer semestre de 1997, con el objetivo de determinar qué actitudes y conocimientos tienen los estudiantes de los semestres XII y XIII, correspondientes al año de práctica (Internado), sobre la menopausia.

Partimos de la base de que la historia personal del individuo limita sus actitudes y estas, a su vez, condicionan el interés y las destrezas que los estudiantes puedan desarrollar hacia un tema determinado. De esta manera, los objetivos fueron múltiples pero, en lo concreto, nos propusimos sondear el nivel de conocimiento científico-práctico, para enfrentar médicamente el tema de la menopausia y poner a prueba la hipótesis de que tal nivel de conocimiento, se relaciona con las actitudes y se asocia además, con la carga cultural del individuo.

\section{Materiales y métodos}

Se aplicó una encuesta a los 128 estudiantes de Internado de la Facultad de Medicina de la Universidad de Antioquia, en el primer semestre de 1997. La información se recolectó por medio de un instrumento que contenía una encuesta de carácter social, un examen de conocimientos cientifico-técnicos y una escala Lickert sobre actitudes frente a la menopausia.

La primera parte de la encuesta permitió describir las variables socioculturales: sexo, edad, lugar de nacimiento del encuestado y de sus padres, lugar de residencia, personas con quienes comparte su residencia y grado de escolaridad de los progenitores. Para evaluar los conocimientos, se realizaron 14 preguntas en forma de test, sobre conocimientos básicos y clínicos acerca de la menopausia. Las preguntas fueron seleccionadas del texto básico de medicina interna, recomendado a los estudiantes que cursan el $7^{\circ}$ semestre (12); y se calificaron en una escala de 0-5.

La escala Lickert contenía 28 items, dirigidos a la medición de actitudes en tres aspectos principales: el primero, indagaba sobre la posibilidad de que las relaciones sexuales en la posmenopausia fueran placenteras; el segundo aspecto fue sobre la actitud frente al uso de terapias, hormonales o sicológicas, para tratar signos y síntomas considerados molestos en el proceso de la posmenopausia; y el tercero, sobre el concepto de las relaciones sociales de la mujer en la posmenopausia. Para la evaluación, se utilizó la técnica estadística de los percentiles, así: de $0 . .40$ se consideró actitud baja, de 41 a 60 actitud media y de 61 a 99 actitud alta.

\section{Resultados}

Resultados de la Encuesta Sociocultural. La distribución porcentual que presentaron las variables sociodemográficas fue la siguiente: el $43 \%$ eran mujeres y $57 \%$ hombres; el $62,5 \%$ tenían entre 20 y 25 años, el 24,2\% entre 26 y 29 años y el $11 \%$ más de 30 años. El 81,3\% nacieron en la ciudad, el 15,6\% en pueblo y el $1,6 \%$ en el campo. El $30,5 \%$ de sus padres tenían origen en la ciudad, el 50,8\% en el pueblo y en el campo el $17,2 \%$; las madres por su parte, nacieron en la ciudad el 39,8, en el pueblo el $46,9 \%$ y en el campo el $11,7 \%$.

El lugar de residencia de los encuestados a lo largo de la vida se distribuye así: en la infancia, el 76,6\% vivieron en la ciudad, $16,4 \%$ en el pueblo y $5,5 \%$ en el campo; en la adolescencia, el $82,8 \%$ vivieron en la ciudad, $10,9 \%$ en el pueblo y $3,9 \%$ en el campo; en la juventud, el $89,1 \%$ 
vivieron en la ciudad, 7,8\% en el pueblo y $1,6 \%$ en el campo; finalmente, en la actualidad, el 94,5\% viven en la ciudad, 3,9\% en el pueblo y $0,8 \%$ en el campo.

En cuanto al estilo de vida familiar, se encontró que el 5,5\% viven solos, mientras que el resto viven con familiares como padre, madre, hermanos y hermanas. Respecto del estado marital, el $92,2 \%$ son solteros, e13,1\% casados, el 2,3\% separados y el $0,8 \%$ viven en unión libre.

El perfil académico de los padres fue el siguiente: el 91,4\% de los padres terminaron primaria, $65,5 \%$ terminaron secundaria, $36,7 \%$ terminaron estudios universitarios y $13,3 \%$ terminaron un postgrado. Las madres, el 95,3\% terminaron primaria, 67,2\% terminaron secundaria, $25,8 \%$ terminaron un programa universitario y $10,9 \%$ terminaron postgrado.

En cuanto a las características de las instituciones donde los encuestados recibieron su educación, e $47,7 \%$ terminaron primaria en escuela pública y el $47,7 \%$ en escuela privada; de las cuales un $24,2 \%$ eran de orientación religiosa. La secundaria fue realizada en el $39,8 \%$ de los casos en institución pública, en el $39,8 \%$ en institución privada y, en este nivel, el 31,3\% pertenece a instituciones de orientación religiosa.

Resultados de la Encuesta de Actitudes. En lo que se refiere a relaciones sexuales placenteras durante y después de la menopausia: eI $43,6 \%$ se situaron en el percentil bajo (0-40), $27,7 \%$ en el percentil medio (41-60) y $28,56 \%$ en el percentil alto (61-99). Sobre el uso de terapia hormonal o psicológica, el $43,6 \%$ presentó actitud baja hacia su uso, 22,2\% media y 40,5\% alta y frente a los cambios en las relaciones sociales durante y después de la menopausia, un $44,4 \%$ se situó en el percentil bajo, un $22,2 \%$ en el mediano y un $33,2 \%$ en el alto.

Resultados de la Encuesta de Conocimientos CientíficoTécnicos. Los resultados globales muestran, para el grupo, una nota promedio de $2,85 \pm 0,83$ sobre un puntaje máximo de 5.0. El 56,24\% de los estudiantes, obtuvo una nota menor o igual a 2,85 (dicho en otros términos "perdieron el examen"). El $43,74 \%$ de los encuestados "aprobaron el examen" y un 10,93\% de ellos, acertaron en 11 o más de las respuestas (puntaje igualo mayor a 4 sobre 5).

Relación entre Actitudes y Conocimientos. Las tablas 1 y 2 , muestran cómo actitudes y conocimientos se correlacionaron positivamente, tanto en hombres como en mujeres.

\section{Discusión}

La población estudiada estaba constituida por menores de 25 años, con una representación equitativa por sexos y una gran mayoría, tanto ellos como de sus padres, de origen citadino. Esto concuerda con la tendencia demográfica de Colombia, hacia un creciente nivel de urbanización. En cuanto al estado civil de los encuestados, en su gran mayoría resultaron ser, solteros que viven en la casa de sus progenitores.

Los datos sobre el nivel académico resultaron interesantes, el $31,2 \%$ de los padres y madres terminaron una carrera universitaria y el $12,0 \%$ terminaron un posgrado; lo cual puede indicar una tendencia de los jóvenes pertenecientes a élites académicas a buscar la medicina u otras profesiones con alto estatus.

Las actitudes, según Sierra (13), "son disposiciones permanentes de ánimo, producto de un conjunto de convicciones
Tabla 0011 i01

\section{DISTRIBUCION DE LAS ACTITUDES SEGUN LA CALIFICACION OBTENIDA EN EL EXAMEN DE CONOCIMIENTOS CIENTIFICOS EN EL GRUPO DE LAS MUJERES}

\begin{tabular}{|c|c|c|c|c|c|c|c|c|c|}
\hline \multirow{2}{*}{$\begin{array}{c}\text { CONOCTOS } \\
\text { CIENTIFICOS }\end{array}$} & \multicolumn{3}{c|}{$\begin{array}{c}\text { RELAC. SEXUALES } \\
\text { PLACENTERAS }\end{array}$} & \multicolumn{3}{c|}{ USO DE TERAPIAS } & \multicolumn{4}{c|}{$\begin{array}{c}\text { RELACIONES } \\
\text { SOCIALES }\end{array}$} \\
\cline { 2 - 12 } & $\begin{array}{c}\% \\
\text { BAJA }\end{array}$ & $\begin{array}{c}\text { MEDIA } \\
\text { ALTA }\end{array}$ & $\begin{array}{c}\% \\
\text { BAJA }\end{array}$ & $\begin{array}{c}\% \\
\text { MEDIA }\end{array}$ & $\begin{array}{c}\% \\
\text { ALTA }\end{array}$ & $\begin{array}{c}\% \\
\text { BAJA }\end{array}$ & $\begin{array}{c}\% \\
\text { MEDIA }\end{array}$ & $\begin{array}{c}\% \\
\text { ALTA }\end{array}$ \\
\hline $\begin{array}{c}\text { CALIFICACION } \\
\text { BAJA }\end{array}$ & 37.5 & 50 & 12.5 & 25 & 50 & 25 & 62.5 & 0 & 37.5 \\
\hline $\begin{array}{c}\text { CALIFICACION } \\
\text { ALTA }\end{array}$ & 40 & 0 & 60 & 10 & 30 & 60 & 40 & 20 & 40 \\
\hline
\end{tabular}

Tabla $0011 \mathrm{i} 02$

DISTRIBUCION DE LAS ACTITUDES SEGUN

LA CALIFICACION OBTENIDA EN EL EXAMEN DE CONOCIMIENTOS CIENTIFICOS EN EL GRUPO DE LOS HOMBRES

\begin{tabular}{|c|c|c|c|c|c|c|c|c|c|}
\hline ACTITUDES & $\begin{array}{l}\text { RELS } \\
\text { PL }\end{array}$ & $\begin{array}{l}\text { C. SEXU } \\
\text { CENTE }\end{array}$ & $\begin{array}{l}\text { ALES } \\
\text { RAS }\end{array}$ & & DE TER/ & IAS & & $\begin{array}{l}\text { ELACION } \\
\text { SOCIALE }\end{array}$ & \\
\hline $\begin{array}{l}\text { CONOCTOS } \\
\text { CIENTIFICOS }\end{array}$ & $\%$ & MEDIA & $\begin{array}{c}\% \\
\text { ALTA }\end{array}$ & $\begin{array}{l}\% \\
\text { BAJA }\end{array}$ & $\begin{array}{c}\% \\
\text { MEOIA }\end{array}$ & ALTA & $\begin{array}{c}\% \\
\text { BAJA }\end{array}$ & MEDIA & $\begin{array}{c}\% \\
\text { ALTA }\end{array}$ \\
\hline $\begin{array}{l}\text { CALIFICACIONN } \\
\text { BAJA. }\end{array}$ & 50 & 0 & 50 & 50 & 25 & 25 & 75 & 0 & 25 \\
\hline $\begin{array}{l}\text { CALIFICACIÓN } \\
\text { ALTA }\end{array}$ & 25 & 25 & 50 & 0 & 75 & 25 & 25 & 0 & 75 \\
\hline
\end{tabular}

y sentimientos, que condicionan la expresión y la acción". El dominio de las actitudes comprende por tanto, tres aspectos: uno ideológico, otro sentimental (simpatías y antipatías) y uno más, el reactivo, que impulsa a pensar y a obrar en concordancia. En el mismo orden de ideas, López (14) sostiene, que las actitudes son adquiridas, permanecen implícitas, son relativamente estables y susceptibles de medición a través de índices semicuantitativos. Zimbardo (15) afirma, por su parte, que muchas de las creencias, actitudes y conductas están determinadas en su mayoría, por grupos relevantes a nuestras vidas: la familia, los amigos, la escuela, etc. Los grupos definen lo socialmente correcto y exigen adherencias mediante recompensas, ame- nazas, ostracismo y otras. Finalmente, Heller(16) lo señala en otros términos, desde una perspectiva antropológica, cuando sostiene que la esencia humana no es el punto de partida, ni el núcleo en el que se superponen las influencias sociales, sino un resultado de la relación activa del individuo, desde su nacimiento, con el mundo.

En esta investigación la distribución de las variables sociales fue relativamente homogénea, lo que no permitió hallar las correlaciones correspondientes, como se lo había propuesto el grupo de investigadores. Sin embargo, la relación hallada entre los tres dominios de actitudes y conocimientos, sí coincide con la hipótesis de trabajo, en cuanto que tanto hombres como mujeres, demostraron una actitud baja cuando el resultado del examen de conocimiento fue bajo y viceversa.

Los resultados de la encuesta de conocimientos muestran, de manera global, una deficiencia conceptual. Esto se considera preocupante ya que los internos son el grupo de estudiantes de medicina de último año, próximos a iniciar su práctica médica general, la cual incluirá el creciente grupo de mujeres en la postmenopausia. Sin embargo, como se mencionó anteriormente, los resultados no son muy diferentes al estudio inglés $(9,10)$; específicamente, es interesante resaltar que el $84 \%$ de nuestros internos contra sólo un $50 \%$ de los médicos del grupo del estudio de la referencia, consideran, correctamente, que el reemplazo con estrógenos disminuye el riesgo cardiovascular de las mujeres en la posmenopausia. 
De las preguntas y respuestas a nivel individual, consideramos conveniente resaltar las siguientes: únicamente el $41 \%$ de los encuestados consideró correcto que las gonadotrofinas se encuentran elevadas en la mujer en estado postmenopáusico. Este es un concepto diagnóstico importante, cuyo desconocimiento puede llevar a la toma de determinaciones terapéuticas equivocadas. En cuanto a la TRH en mujeres histerectomizadas, el $53 \%$ de los encuestados consideró erróneamente, que se requiere la adición de progesterona. Esto podría explicar tendencias hacia la "sobreformulación", por falta de claridad conceptual sobre los riesgos reales de la terapia de reemplazo hormonal y la forma de evitarlos.

Llama la atención, que el $42 \%$ de los encuestados identificó al grupo de mujeres obesas de raza blanca, como el grupo con más alto riesgo de presentar osteoporosis postmenopáusica, colocándolo por encima del grupo de mujeres delgadas de raza blanca que son el grupo de mayor riesgo. Esto puede estar relacionado con estereotipos culturales, que a través de los medios de comunicación, presentan a la mujer delgada como ideal estético y de salud; o podría deberse a una extrapolación de otras situaciones como diabetes o hiperlipidemia, en las cuales la obesidad, sí puede representar un factor de riesgo adicional.

El 87,5\% de los encuestados consideró, en forma correcta, que no sería aconsejable reconiendar estrógenos a mujeres en la posmenopausia, con antecedentes de cirugía por carcinoma de mama hormonodependiente; lo que indica que se tiene identificados a los estrógenos, como hormona relacionada con el cáncer ginecológico(17).

Diferentes grupos de inyestigadores han demostrado que la baja frecuencia en la utilización de terapia de reemplazo hormonal (TRH), guarda relación tanto con los conocimientos y las actitudes del médico $(8,9,10)$, como con los temores y expectativas de las pacientes $(17,18)$.

El análisis de los resultados sobre los conocimientos básicos y clínicos de este grupo de internos, con respecto a la mujer en la menopausia y el climaterio, indica que hay deficiencias específicas en algunas áreas. Esta deficiencia debería ser atendida por la Universidad con cambios en la aproximación pedagógica, empezando por transformaciones de actitud y sensibilidad de todos los profesores -no solamente de los ginecólogos- tendientes a formar al futuro médico en forma equilibrada y teniendo en cuenta la importancia que el tema del climaterio tiene para la salud individual y comunitaria; especialmente ahora, cuando la dinámica demográfica nos está mostrando cambios fundamentales en cuanto al aumento de la expectativa de vida.

Parece que la formación a lo largo de 7 años de estudios en medicina, no está influyendo significativamente en el nivel conceptual con respecto a la menopausia y el climaterio, si se tiene en cuenta que el promedio obtenido no es muy diferente del de una respuesta al azar. Queda claro también que los estudiantes con mejores niveles de conocimientos, tienen una actitud más positiva hacia la mujer en la posmenopausia; o a la inversa, porque de esta investigación no podría definirse cuál es la causa y cuál el efecto; pero sería difícil negar que la influencia de estos factores es mutua y a la vez compleja.

\section{Agradecimientos}

A los estudiantes que contribuyeron con su información y a la Universidad de Antioquia (CODI), por su financiación de la línea de investigación en sexualidad.

\section{BIBLIOGRAFIA}

1. Botella Lussia, J. "La edad crítica", Epidemiología del climaterio. Edit Salvar, Barcelona, Espaila. 1990; 51-61.

2. Departamento Nacional de Planeación. Indicadores Demográficos

3. 1995. En la "Encuesta Nacional de Demografía y Salud. Profamilia. 1995; Cap. 1 y 7.

3. Chiape de V., María L. Demografía Actual. Proyecciones sobre la menopausia y el climaterio. Revista Colombiana de Menopausia. 1995; 1: 51-56.

4. Ettinger B, Genant $\mathrm{H}$. and Conn CE. Long term estrogen replacement therapy prevents bonne loss and fractures. Ann Int Med 1985; 102: 319-324.

5. Stampfer MJ, Graham A, Colditz MB, Willett WC, Manson JE, Rosner B, et al. Postmenopausal estrogen therapy and cardiovascular disease: tenyear follow-up from the nurses health study. N engl J Med 1991; 352: 756762.

6. Takeyoshi Ohkura. Kurnhiro, I. Departamento de Biología Celular, Univ. de Texas. Menopause. 1994; 1: 125-130.

7. Ettinger B, Friedman GD, Bosh T, Quesen Beny CP. Reduced mortality associated with long-term postmenopausal estrogen therapy. Obstet Gynecol 1996; 87: 6-12.

8. Raunikar VA. Compliance with hormone replacement therapy: are women receiving the full impact of hormone replacement therapy preventive health benefits? Women's Health Issues 1992; 2: 75-82.

9. Barlow DH, Brocke JA, Rees CMD. Study of general practice consultations and menopausal problems. Br Med J. 1991; 302: 274-276.
10. Bryce FC, Lilford RJ. General Practitioners'use of Hormone- Replacement Therapy in Yorkshire. Eur J Obstet Gynaecol. 1990; 37: 55-61.

11. Pardo Vargas, F. Editorial Revista Colombiana de Obstetricia y Ginecología; 3:2. Mayo-Agosto 1997.

12. Carr BR, Eilson JD: Disorders of the ovary and female reproductive tract, in Harrison's Principies of Internal Medicine, 13th ed, K J Isselbacher, E Braunwald, JD Wilson, JB Martin, AS Fauci, DL Kasper (eds). New York, Mcgraw-Hill, 1994; 2017-2036 13.

13. Sierra Bravo, Restituto. Escalas de las Ciencias Sociales en Técnicas de Investigación Social y Ejercicios. Madrid, Paraninfo, 1985; 339.

14. López H. Creencias y aptitudes. El cambio social, en Socio- lingüística. Madrid. Ed. Gredos, 1989; 233.

15. Zimbardo, Philip G, Ebbe B. Ebbesen y Christina Maslasch. Examen de las teorías de la actitud y el cambio de la conducta, en: "Influencias sobre las actitudes y modificación de conducta". Introducción al método, la teoría y las aplicaciones del control social y el poder personal. Bogotá. Fondo educativo interamericano. 1982; 41.

16. Heller, Agnes. El saber cotidiano, en "Sociología de la vida cotidiana Barcelona. Península; 1977.

17. Hammond ch B. Women's concerns with hormone replacement therapy complianceissues. Fertility and sterility. 1994; 62(suppl 2): 157s-160s.

18. Ryan PJ, Harrison R, Blake GM, Fogelman 1. Compliance with hormone replacement therapy (HRT) after screening for postmenopausal osteoporosis. Br J Gynaecol. 1990; 75, suppl 4: 77s-83s. 